\title{
俞
}

\section{Automated mechanical ventilation using Adaptive Support Ventilation versus conventional ventilation including ventilator length of stay, mortality, and professional social aspects of adoption of new technology.}

Ronald R. Sanderson RRT, Denise Whitley RRT, Christopher Batacan BS

DOI: https://doi.org/10.53097/JMV.10021

Cite: Sanderson RR, Whitley D, Batacan C. Automated mechanical ventilation using Adaptive Support Ventilation versus conventional ventilation including ventilator length-of-stay, mortality, and professional social aspects of adoption of new technology. J Mech Vent 2020; 2(2):48-52.

\footnotetext{
Abstract

Background

Automation of mechanical ventilation allows for reduction of variation in patient management and has the potential to provide increased patient safety by strict adherence to computer driven ventilator protocols.

Methods:

A retrospective, observational study compared a group of 196 of general ICU patients managed exclusively on automated mechanical ventilation, adaptive support ventilation (ASV), to another group of 684 managed by usual, non-automated mechanical ventilation (No ASV). The data was collected in a unique access database designed to collect data for assessment of mechanical ventilation outcomes in a small medical center ICU.

Results:

The length of ventilator stay was non-significant between both groups, $(81.7 \pm 35.2$ hours $)$ in the ASV group; vs. $(94.1 \pm 35.1$ hours $)$ in the No ASV. Percent mortality was significantly less in the ASV group, $8.6 \%$ compared to $27.3 \%$ in the No ASV.

Conclusion:

Automated ventilation appears to be a safe ventilator strategy; however, cause effect relationships cannot be determined without further, more sophisticated studies.
}

Keywords: Closed loop ventilation, ASV, Ventilator length of stay, Percent minute ventilation

Authors

1. Ronald R. Sanderson, RRT, DrPH, AE-C. Respiratory center of Hawaii. Hawaii, USA

2. Denise Whitley BS, RRT, ACCS. Adventist Health Castle, Kailua, Hawaii, USA

3. Christopher Batacan, BS, MCP, Hawaii, USA

Corresponding author: Ron R. Sanderson: ronsan65@gmail.com

Conflict of interest/Disclosures: None

Funding: None

Journal of Mechanical Ventilation 2021 Volume 2, Issue 2

This open-access article is distributed under the terms of the Creative Commons Attribution Non-Commercial License (CC BY-NC) (http://creativecommons. org/licenses/by-nc/4.0/), which permits reuse, distribution and reproduction of the article, provided that the original work is properly cited and the reuse is restricted to 


\section{Introduction}

Automation of mechanical ventilation is a needed addition to the critical care environment. Intelligent, automatic, breathto-breath adjustment of the patient/ventilator interface by ventilation management protocols imbedded in the ventilator microprocessor is intuitively advantageous as contrasted to ventilator patient management by ICU staff at longer intervals as much as every two to four hours. Numerous reports of closed-loop ventilation are present in the literature. $1,2,3,4,5,6,7$

The more widely available options for "closing the loop" are called dual control systems where the operator typically specifies a tidal volume goal, and the ventilator microprocessor monitors tidal volume for decision making while adjusting inspiratory pressure to keep the tidal volume within specified limits. ${ }^{8,9}$ The patient is assured tidal volume in pressure control modes or during pressure supported spontaneous breaths. The microprocessor either increases or reduces inspiratory pressure in the face of changing lungthoracic compliance and airway resistance to meet the tidal volume goal. Runway inspiratory pressure is avoided with standard, redundant high pressure alarm systems.

A number of "intelligent" or "smart" systems with variable degrees of automation from full automation of the ventilation function to simple aids in ventilator setup are available on more sophisticated mechanical ventilators. These are often misinterpreted as modes of ventilation when they are better described as ventilator protocols imbedded in the ventilator's microprocessor. These protocols move between previously described modes of ventilation to match the patient/ventilator interface. More specifically, precise, well defined, imbedded patient management protocols use physiologic data provided by the ventilator monitor to adjust the ventilator to the patient. Imagine an excellent respiratory care practitioner standing at the patient's bedside continuously assessing the ventilator/patient interface and adjusting the ventilator breathto-breath using a lung protective strategy. An analogy to autopilot for airplanes may be reasonable.

The automated, closed-loop ventilation system used in this group of patients is a very sophisticated and reliable multicontrol system (Adaptive Support Ventilation [ASV], Hamilton Medical Inc., Reno, Nevada) where the ventilator microprocessor monitors six variables used for decision making: tidal volume, respiratory rate, expiratory time constant $\left(\mathrm{RC}_{\mathrm{exp}}=\mathrm{C}_{\text {stat }} / \mathrm{R}_{\mathrm{exp}}\right)$ spontaneous breathing, and peak pressure. ASV makes changes in five parameters: tidal volume, mode, peak pressure, respiratory frequency, and inspiratory time. The ventilator microprocessor does this with input from the operator for the patient's ideal body weight (IBW) $10-200 \mathrm{Kg}$. and desired percentage (50\% - 350\%) of ideal minute ventilation $\left(\% \mathrm{~V}_{\mathrm{E}}\right)$. Oxygenation $\left(\mathrm{FIO}_{2}\right.$ and PEEP) settings were controlled by the operator.

An ideal illustration of this process is placement of a postoperative patient on the ventilator for recovery. The operator sets IBW, $\% \mathrm{~V}_{\mathrm{E}}, \mathrm{FIO}_{2}$, PEEP and alarms, and then attaches the anesthetized patient to the ventilator circuit. The ventilator microprocessor gives a few test breaths while monitoring the patient and analyzing decision-making data. It then establishes tidal volume and respiratory frequency goals and places the patient in a control mode of ventilation (PCV-SIMV) using lung protective strategies. As the patient wakes up and initiates spontaneous respiration the ventilator follows patient changes, breath-to-breath, weaning the patient to spontaneous mode with pressure support and reduces pressure support to ready the patient for extubation. (Figure 1).

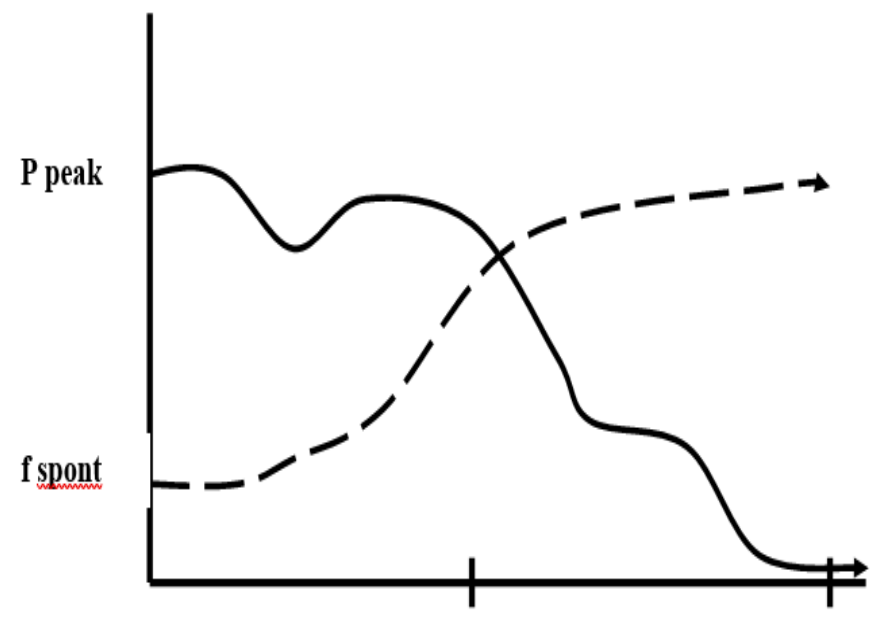

Figure 1. Schematic of ideal ASV Management of a Postoperative Patient's Ventilation

The process is similar for difficult patients in multisystem failure such as ARDS where the ventilator changes ventilation targets as the patient's $\mathrm{RC}_{\mathrm{exp}}$ changes and the patient goes in and out of spontaneous breathing with changing levels of respiratory drive related to level of consciousness or sedation usage. ASV will increase or decrease support as the patient condition changes. It is important to note that $\mathrm{RC}_{\mathrm{exp}}$ is a value representing the patient's lung/thoracic compliance and expiratory airway resistance which is continuously measured by the ventilator. Practically the time constant $(\pi)$ for exhalation can be thought of as exhalation time that will be shorter with low lung/thoracic compliance or will be longer with increased airway resistance. 


\section{Methods}

This is a retrospective study from data collected in a unique Access database constructed to collect data for assessment of mechanical ventilation outcomes in a small medical center ICU over period of five years. 196 patients were managed exclusively on automated ventilation, Adaptive Support Ventilation (ASV) - Hamilton Medical ventilators, while 694 patients received mechanical ventilation using exclusively usual, non-closed-loop technology. The automated protocol on the ventilator uses a clear, consistent algorithm for decision making based on Otis's least Work of Breathing equation ${ }^{10,11}$ and a low tidal volume, lung protective strategy ${ }^{12}$ that creates low priority alarms closely around tidal volume, rate and pressure goals while being backed-up by the standard complement of high and low priority ventilator alarms. (Figure 2).

Ventilator management of both groups was accomplished in the same ICU, with the same staff, same standard of care and managed by the same mix of physicians who are experienced in ventilator patient management. This is a report of a series of patients receiving clinical care in a small hospital of 157 beds. No experimental methods were used, and no study parameters were defined prior to or during data collection. Data was collected similarly on all patients and analyzed retrospectively. This is a convenience sample and excluded all ventilator patients who spent time on both closed-loop technology and non-closed loop technology during the same ventilator stay. The medical center exempted consent as this is a retrospective, observational study, not an experimental study, and no link can be made from the data set to any specific patient. $T$ test of equal variance was used to calculate the difference in VLOS and mortality.

Otis's Least Work of Breathing Equation ${ }^{13}$

$$
f=\frac{\sqrt{1+2 a \times R C e \times(\mathrm{MinVol}-f \times V d) /(V d)}-1}{a \times R C e}
$$

Where:

$\mathrm{a}=2 \pi^{2} / 60$

$\mathrm{RCe}($ Expiratory time constant $)=\mathrm{R}_{\text {exp }} / \mathrm{C}_{\mathrm{rs}}$ (seconds)

$\mathrm{R}_{\exp }=$ Expiratory airway resistance $\left(\mathrm{cmH}_{2} \mathrm{O} / 1 / \mathrm{sec}\right.$. $)$

$\mathrm{C}_{\mathrm{rs}}=$ Compliance, respiratory system $\left(\mathrm{ml} / \mathrm{cmH}_{2} \mathrm{O}\right)$

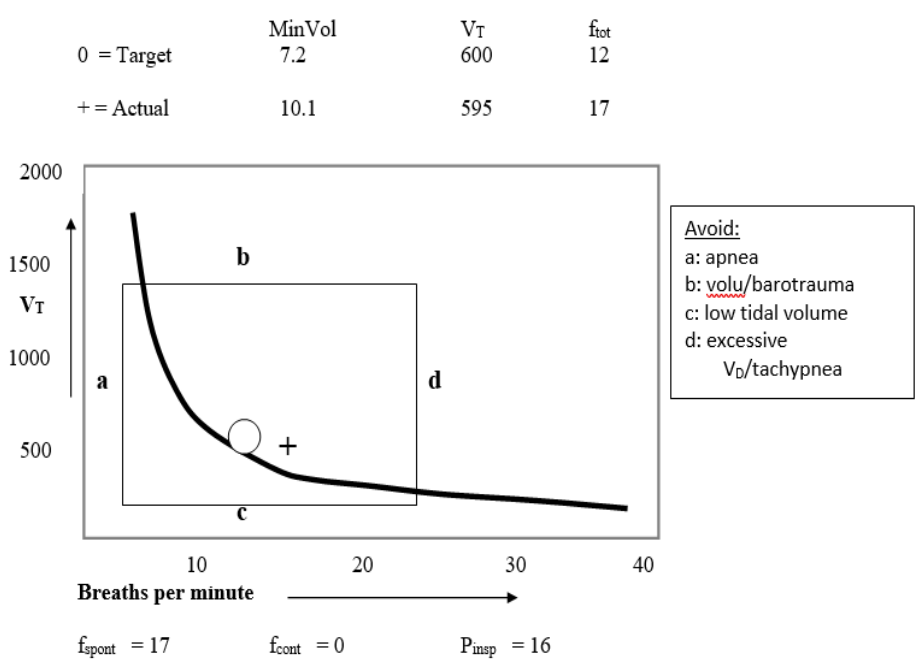

Figure 2. Schematic of the Adaptive Support Ventilation Monitor Screen

\section{Results}

Ventilator length of stay between the two groups were not significant. Ventilator patient mortality on closed-loop ventilation was less than one third (31.5\%) and was statistically significant compared to those on non-closed loop ventilation.

\begin{tabular}{|l|l|l|l|}
\hline & ASV Only & No ASV & P value \\
\hline Number of patients & 196 & 684 & N/A \\
\hline Average hours on ventilator & $80.2 \pm 35.2$ & $92.7 \pm 35.1$ & 0.37 \\
\hline$\%$ Mortality on ventilator & 8.6 & 27.3 & $<0.03$ \\
\hline
\end{tabular}

Table 1. Results of Closed Loop and Non-closed Loop Mechanical Ventilation

These results are subject to bias from multiple sources because of the convenience sample. There may have been practitioner bias in favor of or opposed to closed-loop ventilation. Patient's characteristics, severity of illness was not documented in the database; however, both ASV and nonclosed loop ventilation was used on the full spectrum of ICU patients; however, non-randomly. ICU nurses liked ASV because it reduced nuisance alarms. Respiratory Care Practitioners liked ASV because the patients seemed to be more comfortable and there were less calls to physicians to make changes in physician orders related to tidal volume and respiratory rate. The physicians remain skeptical; however, happily noted the decrease in after-hours calls for ventilator patient care. 


\section{Discussion}

Initial apprehension surrounding adoption of comprehensive closed-loop ventilation included physician concerns about patient safety from barotrauma and volutrauma, ability to assess where the ventilator microprocessor had "taken the patient", the need for practitioners at the bedside and what physician orders are necessary. ${ }^{14}$ Concerns for patient safety were allayed by the presence of low and high priority ventilator alarms. The respiratory care practitioner could assess the precise ventilator configuration breath-to-breath in a monitor screen that consolidates the important variables.

Practitioners at the bedside had additional, new information to assess the patient/ventilator relationship. The ventilator made important adjustments breath-to-breath that were completely impractical for a bedside practitioner even with a hypothetical 1:1 patient ratio. Physicians ordered initial settings $\left(\% \mathrm{~V}_{\mathrm{E}}\right.$, $\mathrm{FIO}_{2}, \mathrm{PEEP}$ ) in addition to any limits/goals for $\mathrm{PaO}_{2}$, and $\mathrm{PaCO}_{2}$. The team treated the automated process as a ventilator protocol that has the fringe benefit of $100 \%$ compliance.

Clinicians without experience of comprehensive closed-loop ventilation often respond, "If we use this why do we need a respiratory care practitioner or intensivist?" The answer is clearly that the respiratory care practitioner and intensivist assess the patient and deliver appropriate respiratory care outside the parameters controlled by the ventilator and specify the level of control provided by the automated protocol. ${ }^{15} \mathrm{In}$ addition, most respiratory care practitioners have accepted automation in blood gas analyzer calibration and running of controls and/or use of feedback from heated wire circuits to humidifiers. Another question is, "How does this perform on the difficult to manage patient?" The closed-loop process seemed to do as well as non-closed-loop ventilation. It seemed to perform well on complex, multi-system failure ARDS patients and across the spectrum of ventilator patients seen in a small medical center with an active emergency department. 16

ASV does struggle with tachypnea in the spontaneous breathing patient. ASV has the ability to automatically conduct spontaneous breathing trials if sedation is lightened and $\mathrm{PaCO}_{2}$ is appropriate. Positive aspects of ASV for postoperative cardiac surgery patients has been reported. ${ }^{17}$ Reduction of staffing and ICU costs have been described with while using ASV for discontinuation of mechanical ventilation in difficult to wean patients without continuous support of respiratory therapists or intensive care specialists. ${ }^{18}$

Institute of Medicine findings on medical error and subsequent pressure from many sectors to reduce medical error by automating processes make ventilator imbedded protocols appear to be an attractive direction for investigation and development. ${ }^{19}$

We suspect that automation of mechanical ventilation has the potential to become the standard of ventilator care in the future and will eclipse ventilator care as we currently know it; because automation increases good decision making and reduces error.

\section{Conclusion}

Automated ventilation appears to be a safe ventilator strategy in terms of ventilator LOS and mortality; however, no causeand-effect relationships can be inferred from this retrospective, non-random, observational studies. Further study in larger groups of patients to evaluate decreased mortality and length of stay must be done to validate the performance of closed loop ventilation in varied patient environments.

\section{References}

1. Sulzer CF, Chiolero R, Chassot PG, et al. Adaptive support ventilation for fast tracheal extubation after cardiac surgery: a randomized controlled study. Anesthesiology 2001; 95(6):1339-1345

2. Moradian ST, Saeid Y, Ebadi A, et al. Adaptive Support Ventilation reduces the incidence of atelectasis in patients undergoing coronary artery bypass grafting: A randomized clinical trial. Anesth Pain Med. 2017; 22;7(3):e44614.

3. Petter AH, Chiolero RL, Cassina T, et al. Automatic "respirator/weaning" with adaptive support ventilation: the effect on duration of endotracheal intubation and patient management. Anesth Analg 2003; 97(6):1743-1750.

4. Campbell RS, Sinamban RP, Johannigman JA, et al. Clinical evaluation of a new closed loop ventilation mode: adaptive supportive ventilation (ASV). Critical Care 1999; 3(Suppl 1): P038.

5. Van der Staay M, Chatburn RL. Advanced modes of mechanical ventilation and optimal targeting schemes., Intensive Care Med Exp 2018; 22;6(1):30.

6. Linton DM, Potgieter PD, Davis S, et al. Automatic weaning from mechanical ventilation using an adaptive lung ventilation controller. Chest 1994; 106:1843-1850.

7. Laubscher TP, Frutiger A, Fanconi S, et al. The automatic selection of ventilation parameters during the initial phase of mechanical ventilation. Intensive Care Med 1996; 22(3):199207. 
8. Schaublin J, Derighetti M, Feigenwinter P, et al. Fuzzy logic control of mechanical ventilation during anaesthesia. British Journal of Anaesthesia 1996; 77: 636-641.

9. Branson RD, Johannigman JA, Campbell RS, et al. ClosedLoop Mechanical Ventilation. Respir Care 2002; 47(4):427451.

10. Sanderson R, Wheatley D, Soca K, et al. Comparison of Ventilator Stay and Mortality between Closed-Loop/Nonclosed Loop Mechanical Ventilation", Respir Care 2005; $50(11)$.

11. Quan SF, Parides GC, Knoper SR. Mandatory minute volume (MMV) ventilation: an overview. Respir Care 1990; 35(9):898-905.

12. Sviri S, Bayya A, Levin P. et al. Intelligent ventilation in the intensive care unit. J Crit Care 2012; 28(1):6-12.

13. Otis AB, Fenn WO, Rhan H. Mechanics of Breathing in Man, J Appl Physiol 1950; 2:592-607.

14. Gattinoni L, Chiumello D, Russo R. Reduced tidal volumes and lung protective ventilatory strategies: Where do we go from here? Current Opinion in Critical Care 2002; $8(1): 45-50$.

15. Vogelsang, H, Uhlig T. A prospective randomized study on clinical and economical aspects of closed loop control and common weaning protocols after cardiac surgery. Critical Care 2007; (Suppl 2):173.

16. Wheatley D, Young, K. Adaptive support ventilation. What is it? Beneficial or not? J Mech Vent 2020; 2(1):34-44.

17. Marini JJ, Crooke PS, Truwit JD. Determinants and limits of pressure-preset ventilation: a mathematical model of pressure control, Journal of Applied Physiology 1989; 67:1081-1092.

18. Otis AB. The work of breathing. In: Fenn WO and Rahn $\mathrm{H}$, editors. Handbook of Physiology. Section 3: Respiration. Washington DC: American Physiological Society; 1964:463476.

19. Linton DM, Renov G, Lafair J, ET AL. Adaptive Support Ventilation as the Sole Mode of Ventilatory Support in Chronically Ventilated Patients, Critical Care and Resuscitation 2006; 8:11-14. 


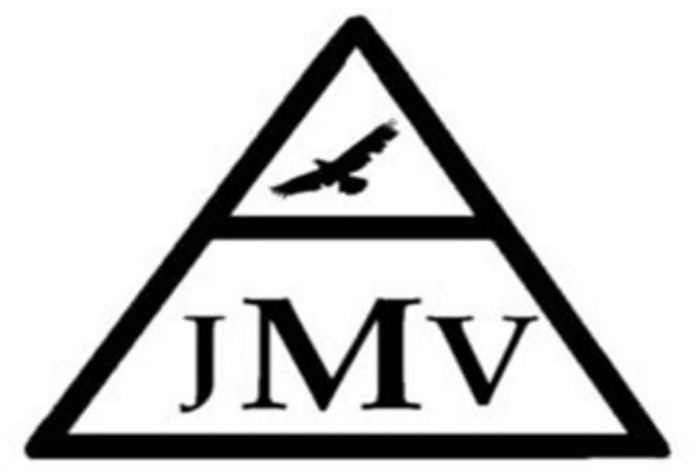

Journal of Mechanical Ventilation

Submit a manuscript

https://www.journalmechanicalventilation .com/submit-a-manuscript/

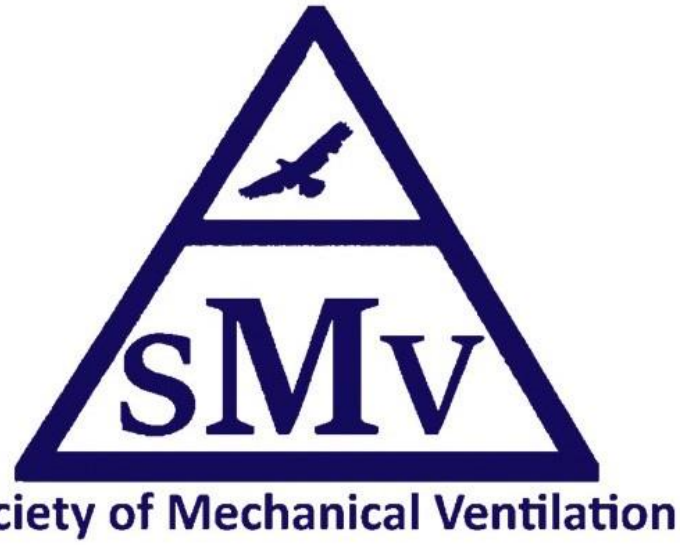

Free membership

https://societymechanicalventilation.org /membership/ 